\title{
The task 3 of forming a network on the sphere from the circles of the same radius
}

\author{
Vladimir Travush ${ }^{1}$, Vasily Antoshkin ${ }^{2, *}$, and Mariya Chorina $^{2}$ \\ ${ }^{1}$ Russian Academy of Architecture and Construction Sciences, Bolshaya Dmitrovka Str. 24, Building \\ 1, 107031, Moscow, Russia \\ ${ }^{2}$ Ogarev Mordovia State University, Bolshevistskaya Str. 68, 430005, Saransk, Republic of Mordovia, \\ Russia
}

\begin{abstract}
: one of the methods of formation of geometric networks of arches of the same radius using regular spherical polyhedra is Investigated. The conditions of the task of placing the specified network on the sphere are set. The criterion for evaluating the effectiveness of solving the problem is the minimum number of standard sizes of segments of the dome arches, the possibility of using technologies of enlarging assembly. The solution of one variant of the problem of placing a geometric network on a spherical cube and, accordingly, on a sphere is given. Placement on the sphere of arches of one radius, different from placement in the form of meridians, has an effective solution in the form of a network with minimal dimensions of arch segments and nodes of paired arches formed on the basis of circles of identical radii formed on the basis of regular spherical polyhedra. The problem is solved by constructing an independent framework of arches of the same radius on the basis of paired circles of the same radius in the spherical cube system.
\end{abstract}

\section{Intduction}

The problem of most of the known solutions of geodesic and mesh domes is a large number of different types of nodes connecting elements of shells, sharp angles of connections, different types and complexity of the support nodes, due to the fact that these support nodes are at different levels, as well as the complexity and laboriousness of the implementation of enclosing structures and roofing.

To ensure certainty of the reproduction of the geometry of the domed cover, consequently, improve reliability, to simplify the manufacturing nodes of the frame shell and fabrication of building envelopes and roofs, requires the solution of problems associated with a decrease in the number of mounting elements, the reduction of the material of joints, and formation, by optimizing the geometry of the sphere cuts (eliminating sharp corners), the location of the supports at the same level of sphere, a significant reduction in the number of sizes of nodes elements.

\footnotetext{
* Corresponding author: antovd@mail.ru
} 
As a result of this work, geodesic domes are offered with a specific cuts, which is performed by splitting the faces with large circles along the midpoints of the edges and the centers of the faces of a regular spherical polyhedron, for example, a cube or an octahedron, etc. [1-9]. The first cut of the spherical shell framework is obtained by splitting into spherical elements and shapes, for example, arched arcs, polygons, triangles, etc., paired circles of the same radius parallel to the specified large circles passing through the middle of adjacent ribs. The second cut of the frame is formed by paired circles of the same radius, parallel to said large circles passing through the middle of the ribs and through one rib. The third cut is formed by paired circles parallel to large circles passing through the vertices of the faces of the corresponding regular spherical polyhedron. The fourth, fifth, sixth and seventh cuts of the frame can be formed by combining the first and second, second and third, first and third, as well as all at once cutting options, if necessary and appropriate.

In the considered geodesic dome on the basis of a regular spherical polyhedron (cube or octahedron), it is possible to place a network with minimum standard sizes of arch segments and with nodes of paired arches formed on the basis of circles of the same radii and regular relative to the faces of the polyhedron, which will provide an effective arrangement of common and support nodes [1-13].

Figure 1 shows the correct spherical polyhedron is a cub. Vertex of the faces is denoted as $\mathrm{O}$, the center of face $\mathrm{K}$, and the radius of circles of the same radius as $r$. If analyze the possibility of such a variant of the geometric network on the sphere, when the arc forming sides of figures in the form of a triangle and of a square are equal, you should choose an appropriate schema for the task. Pluses for constructing radiuses (figure 1), should be select the centers $\mathrm{O}$ the correct spherical cube, and for a geometric network should show the scheme in figure 1, where arcs $r$ cut off at the centers of faces and ribs of faces of the arc $o$ and the arc $\boldsymbol{c}$, which determine the position of the major figures of the network from a paired of arches of the same radius.

\section{Decision}

In the scheme of figure 1 shows the placement of paired circles of the same radius [1-13] on the basis of a spherical cube. Given the known location of the centers of the circles in the centers of opposite faces, the problem of forming a geometric network on a sphere with the centers of nodes located on the circles parallel to the equator circle (i.e. at one mark) is reduced to solving a system of equations for the spherical triangles OOK and OKF, given in figure 1.

We present the known parameters of constructions from a network of paired circles on a spherical face $\mathrm{OOOO}$ of a regular hexagon (cube) inscribed in a sphere, a faces in the form of a spherical square. We show by our solutions that a simple partition with two sizes of arcs in a system of paired circles is possible for a cube.

By the condition of the task when paired circles are composed of a repeating arcs of length $2 \mathrm{a}$ and $2 \mathrm{~b}$, it turns out that the internal angle for a spherical cube at the zenith of the consider triangle in figure $1-\mathrm{OOK}=2 \mathrm{~A}+2 \mathrm{~B}=60^{\circ}$, that is the internal angles $\mathrm{A}+\mathrm{B}=30^{\circ}$, they pull together arcs equal to $a$ and $b$. The internal angle of the triangle OKF as central corner of the square at the center of the cube face subtending arc is $\mathrm{r}$ equal to $135^{\circ}$, it, respectively, with the sides of the $o$ and $k$; and the central angle of a regular triangle on the top of the face is OTE $=60^{\circ}$, it - with sides of $c$ and $t$. Here, on the face of the cube, there are also notations: $r$ - the radius of the circle parallel to the equator from the pole $\mathrm{O} ; d$ - the height of the triangle EOF; $e$ - the arc CT at the top of the face - all in the form of polar angles. 
Using The known neper expressions [15] for the sides and angles of rectangular spherical triangles, we obtain a system of equations (1 and 2):

$$
\sin k=\frac{\sin a}{\sin 45^{\circ}} ; \frac{\sin a}{\sin 60^{\circ}}=\sin t ;
$$

$\cos r=\cos k \cos o+\sin k \sin o \cos 135^{\circ} ; \cos r=\cos t \cos c+\sin t \sin c \cos 60^{\circ}$.

From two equations (1) obtain

$$
\begin{aligned}
& \sin k=\frac{\sin a}{\sin 45^{0}}=\sqrt{2} \sin a, \sin t=\frac{\sin a}{\sin 60^{0}}=\sqrt{\frac{4}{3}} \sin a, \\
& \cos k=\sqrt{1-2 \sin ^{2} a}, \quad \cos t=\sqrt{1-\frac{4}{3} \sin ^{2} a} .
\end{aligned}
$$

Substitute the values of the internal angles (3) into the transformed equation (2)

$$
\begin{gathered}
\cos t \cos c+0,5 \sin t \sin c=\cos k \cos o-\sqrt{0,5} \sin k \sin o \\
\cos t \cos c-\cos k \cos o=-0,5 \sin t \sin c-\sqrt{0,5} \sin k \sin o
\end{gathered}
$$

Substitute from formulas (3) into the resulting equation (4)

$$
\begin{aligned}
& \cos c \sqrt{1-\frac{4}{3} \sin ^{2} a}-\cos o \sqrt{1-2 \sin ^{2} a}=-\sqrt{\frac{1}{3}} \sin c \sin a-\sin o \sin a \\
& \cos ^{2} c\left(1-\frac{4}{3} \sin ^{2} a\right)-2 \cos c \cos 0 \sqrt{\left(1-2 \sin ^{2} a\right)\left(1-\frac{4}{3} \sin ^{2} a\right)}+\cos o\left(1-2 \sin ^{2} a\right)=\left(-\sqrt{\frac{1}{3}} \sin c-\sin o\right)^{2} \sin ^{2} a ; \\
& 2 \cos c \cos o o \sqrt{\left(1-2 \sin ^{2} a\right)\left(1-\frac{4}{3} \sin ^{2} a\right)}=\cos ^{2}\left(1-2 \sin ^{2} a\right)+\cos ^{2} c\left(1-\frac{4}{3} \sin ^{2} a\right)-\left(\sqrt{\frac{1}{3}} \sin c+\sin ^{2}\right)^{2} \sin ^{2} a ; \\
& 4 \cos ^{2} c \cos ^{2} o\left(1-2 \sin ^{2} a\right)\left(1-\frac{4}{3} \sin ^{2} a\right)=\left(\left(\cos ^{2} o+\cos ^{2} c\right)-\left(2 \cos ^{2} o+\frac{4}{3} \cos ^{2} c+\left(\sqrt{\frac{1}{3}} \sin c+\sin o\right)^{2}\right) \sin ^{2} a\right)^{2} ; \\
& 4 \cos ^{2} c \cos ^{2} o-4\left(\frac{4}{3} \cos ^{2} c \cos ^{2} o+2 \cos ^{2} c \cos ^{2} o\right) \sin ^{2} a+\frac{32}{3} \cos ^{2} c \cos ^{2} o \sin ^{4} a=\left(\cos ^{2} o+\cos ^{2} c\right)^{2}- \\
& -2\left(\cos ^{2} o+\cos ^{2} c\right)\left(2 \cos ^{2} o+\frac{4}{3} \cos ^{2} c+\left(\sqrt{\frac{1}{3}} \sin c+\sin o\right)^{2}\right) \sin ^{2} a+\left(2 \cos ^{2} o+\frac{4}{3} \cos ^{2} c+\left(\sqrt{\frac{1}{3}} \sin c+\sin o\right)^{2}\right)^{2} \sin ^{4} a \\
& \left(\frac{32}{3} \cos ^{2} c \cos ^{2} o-\left(2 \cos ^{2} o+\frac{4}{3} \cos ^{2} c+\left(\sqrt{\frac{1}{3}} \sin c+\sin o\right)^{2}\right)^{2}\right) \sin ^{4} a-\left(\left(\frac{40}{3} \cos ^{2} c \cos ^{2} o\right)-\right. \\
& -\left(2\left(\cos ^{2} o+\cos ^{2} c\right)\left(2 \cos ^{2} o+\frac{4}{3} \cos ^{2} c+\left(\sqrt{\frac{1}{3}} \sin c+\sin o\right)^{2}\right)\right) \sin ^{2} a+4 \cos ^{2} \cos ^{2} o-\left(\cos ^{2} o+\cos ^{2} c\right)^{2}=0 .
\end{aligned}
$$

To covering the dome of profiled or flat sheet requires bending these sheets by the surface of the sphere due to deformation of the corrugations. For deformation of sheets it is necessary to strengthen strips of a covering with one more pair of arches with radius $\mathrm{c}+\mathrm{t}=$ $=81,950533050196^{\circ}$ without formation of superfluous additional knots (figure 2). The width of the arc between these paired arches $\mathrm{h}=16,098933899608^{\circ}$.

At almost identical schemes in the specified figure 2 the scheme of a - pavilion-canopy with additional pair arches where $h$ - height of a strip consisting of 2 pair arches two radii which divide these arches into almost identical arcs (i.e. distances between pair circles of both frameworks in the form of polar corners) is shown. 


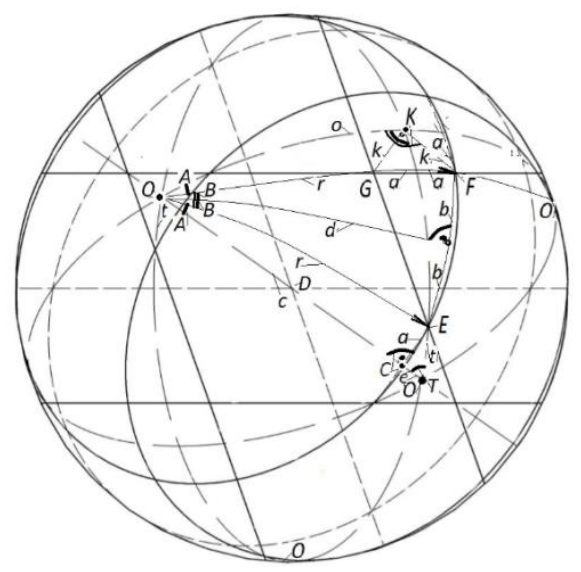

Fig. 1. The schema of a geometric network of circles of the same radius based on the spherical cube; $\mathrm{K}$-center face of the cube on the sphere; $\mathrm{O}$ is the vertices of the faces, and the pole axes of the framework; $r$ is the radius of the circles of a pair of arches.

a)

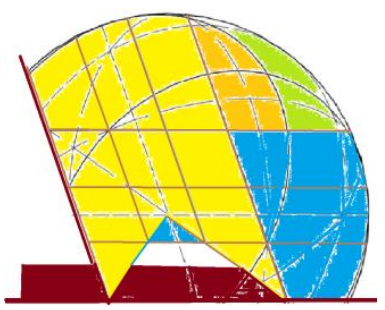

b)

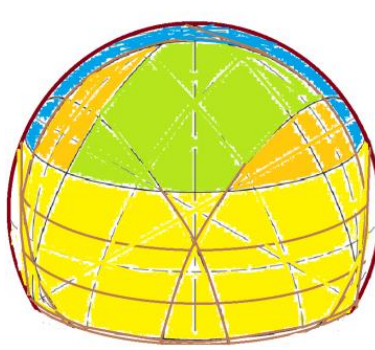

c)

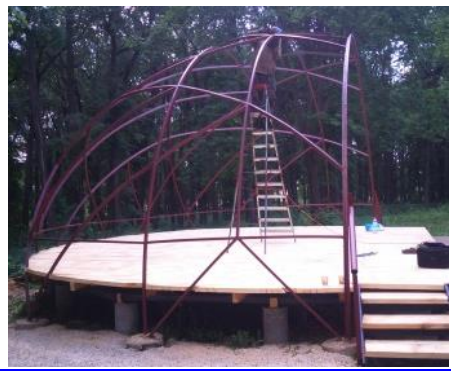

Fig. 2. Canopy scene with a cut of 2 pairs of circles 2 radii of one independent arched framework (poles at the tops) on the basis of a spherical cube: a - side view of the canopy, $\mathrm{b}$ - top view, $\mathrm{c}$ - view of the erected framework of steel tubular paired arches.

The chaotic arrangement of the arches of the combined circumferences of the arches in figures $2 \mathrm{a}, \mathrm{b}, \mathrm{c}$ actually contains two regular simple frameworks of paired arches.

\section{Summary}

The proposed solution of the cuts is the geometric basis of a geodesic dome or dome segment formed by paired circumferences the same radius. It allows you to greatly simplify the solution of all nodes of the shell, in which no more than four elements converge; including support nodes, since the base is made with nodes on the same level. These structures can be assembled from individual arches, and it is also possible to apply technologies of enlarging assemblies of dome elements. Technological solutions such domes provide certainty playback geometry of such a complex shape, simplifies the manufacture of parts of the skeleton of such a sheath, device for covering and roof.

\section{References}

1. V. D. Antoshkin, Academia. Architecture and construction, 1, 133-138 (2017) 
2. V.D. Antoshkin, International Journal for Computational Civil and Structural Engineering, 2(13), 154-160 (2017)

3. V. D. Antoshkin, Regional architecture and engineering, 3 (24), 112-121 (2015)

4. V. D.Antoshkin, S. S. Gudozhnikov, O. I. Perfilieva, I. V. Proceedings of the Thirteenth International scientific technical conference, 12(2), 4-15 (2014)

5. V. D. Antoshkin., V. I. Nikonov, Fundamental research, 11-8, 1669-1673 (2014)

6. V.D. Antoshkin, A.G. Konovalov, Ogarev-Online, 13 (54), 6-9 (2015)

7. V.D. Antoshkin, G.V. Kurbakov, V.S. Bochkin, StredoevropskyVestnik pro Vedu aVyzkum, 83(1), (2015)

8. D. V. Alekseevskii, E.B. Vinberg, D.I. Solodovnikov, Geometry of spaces of constant curvature. Results in science and technology.Current problems in mathematics, Fundamental Directions (VINITI, 1998)

9. V. D. Antoshkin, V. G. Kurganski, Stredoevropsky Vestnik pro Vedu a Vyzkum, 83(2), (2015)

10. V. T. Erofeev, O. V.Startsev, V. D. Antoshkin, S. S. Gudozhnikov, E. G. Smolkin, I.V. Boldin, A.Y. Makhankov, Basic research, 9-12, 2630-2638 (2014)

11. W. Lienhard, Elem. Math, 66 (2), 74-82 (2011)

12. E.F.Yezhov, Y.V. Yurkin, V.D. Antoshkin, V.E.Yezhov, Modern technologies of building materials and structures (Saransk, 2003)

13. B. V. Miryaev, Regional architecture and engineering, 3, 122-125 (2012)

14. A. G. Konovalov, V. D. Antoshkin, Proceedings of the Thirteenth International scientific-technical conference, 14(2), 48-53 (2015)

15. G. A. Korn, T. M. Korn, Mathematical Handbook for Scientists and Engineers, 1151 (2012)

16. Software Scilab 5.4.1 - The free platform for Numerical Computation/ Date Views 06.17.2014 / www. softkumir.ru/index

17. V. D Antoshkin, V. I.Travush, M. V. Gorina, D.V.Antoshkin, Spherical shell with coating, Patent RU No 82685602, 22/04/2019

18. V. I. Travush, V. D. Antoshkin, I. V. Yerofeyeva, D. V. Antoshkin, Team spherical shell, Patent RU No 2564545 07/28/2014

19. V. I. Travush, V. D. Antoshkin, V. T. Erofeyev, S. S.Gudozhnikov, Construction and reconstruction, 6 (50), 36-48(2013)

20. V. I. Travush, V. D.Antoshkin, V. T.Erofeev, MATEC Web of Conferences, 86, 01031 (2016)

21. V. I. Travush, V. D. Antoshkin, V. T. Erofeev, MATEC Web of Conferences, 86, 01032 (2016)

22. V. I.Travush, V. D Antoshkin, MATEC Web of Conferences 106, 02012 (2017)

23. V. I.Travush, V. D. Antoshkin, MATEC Web of Conferences 106, 02016 (2017)

24. V. I. Travush, V. D. Antoshkin, A. Yu. Svyatkina, TPACEE 2018 E3S Web of Conferences, 91, 02011(2018)

25. V. I. Travush, V. D. Antoshkin, A. Yu. Svyatkina, BULLETIN of TOMSK STATE University of ARCHITECTURE and CONSTRUCTION, 6(20), 98-106 (2018) 